\title{
Überlegungen zu einem integrativen Frame-Modell: Elemente, Ebenen, Aspekte
}

\author{
Dietrich Busse
}

\begin{abstract}
Frame-Theorien sind (zumindest aus linguistischer Sicht) kein monolithischer Block. Frame-Theorien, die sich auf Prädikationen (Prädikatsausdrücke, meist: Verben) fokussieren (wie Fillmore und FrameNet), stehen solche gegenüber, die eindeutig Nomen zum Lieblingsgegenstand nehmen (wie Barsalou). Die teilweise erheblichen Unterschiede zwischen Frame-Modellen lassen sich auch durch diese unterschiedliche Fokussierung erklären. Eine für linguistische (insbesondere semantische) Zwecke nutzbare Frame-Analyse muss solche Differenzen jedoch überwinden und ein umfassendes, integratives Modell der Frame-Semantik formulieren. Die wichtigsten Elemente, Ebenen und Aspekte eines solchen integrativen Frame-Modells sollen erläutert werden. Eingeordnet sind die Überlegungen dazu in das, was ich seit einigen Jahren als „linguistische Epistemologie“ bezeichne - im vorliegenden Kontext verstanden als eine umfassende Analyse des verstehensrelevanten Wissens als Teil einer wissensorientierten (linguistischen) Semantik.
\end{abstract}

\section{Einleitung}

Die Geschichte der modernen Linguistik, auch und gerade der linguistischen Semantik, ist eine Geschichte der systematischen Ignorierung bzw. Verdrängung des verstehensrelevanten menschlichen Wissens in seiner ganzen funktional wirksamen Breite in den Theorien und der Methodik dieser Forschungsdisziplin. Im unbewussten Vertrauen auf die eigene muttersprachliche Intuition haben die Forscherinnen und Forscher verdrängt, wie umfassend, wie subtil und wie wenig dem sogenannten „Wortlaut“ der sprachlichen Zeichenketten direkt entnehmbar 
dieses verstehensrelevante Wissen ist. Oder anders ausgedrückt: Geblendet durch theoretische Axiome, die ihren Ursprung meist außerhalb der Sprachwissenschaft im eigentlichen Sinne hatten, und die meist stärker durch theoretische Ideologeme aus Philosophie und mathematisch inspirierter Logik beeinflusst waren und sind als durch genuin einzelsprachbezogene Erkenntnisse, ${ }^{1}$ hat es die Linguistik versäumt, ganz präzise der entscheidenden semantischen Frage näher nachzugehen. Das ist die Frage, in welcher Weise sprachliche Zeichen und Strukturen auf allen Ebenen der Organisation von Sprache denn ihren jeweiligen Beitrag dazu leisten, dass Menschen dasjenige Wissen aktivieren können, das sie benötigen, um den leeren Schall oder die Tinten- oder Pixel-Formationen zu sinnvollen Zeichen und sinnvollen sprachlichen Ausdrücken bzw. kommunikativen Handlungen zu machen.

Man kann in diesem Zusammenhang von einer "Zeichenvergessenheit“ der modernen Linguistik sprechen, die für viele ihrer Gebiete, insbesondere für Morphologie und Syntax, kennzeichnend ist. Aus anderer Perspektive kann man vielleicht auch von einer „Wissensignoranz“ sprechen, $d$. h. einer beständigen Tendenz der modernen Linguistik, dasjenige verstehensrelevante Wissen, das in linguistischen Analysen überhaupt berücksichtigt wird, sukzessive auf ein Minimum zu reduzieren und die tatsächliche Breite und Tiefe des verstehensrelevanten Wissens zu bloßen Kontextfaktoren zu deklarieren - natürlich mit dem Ziel, deren Erforschung aus dem Bereich der „wahren Linguistik“ zu exorzieren.

Im Gegensatz also zum linguistischen Mainstream macht für mich linguistische Semantik und Textanalyse nur als Wissensanalyse einen Sinn. Genauer: als akribische Analyse des Beitrages, den jedes kleine sprachliche Zeichen und Teilzeichen (bis hinunter auf die Ebene der Morphologie), jedes sprachliche Signal, und sei es ein durch Wortstellung oder Satzglied-Stellung gegebenes Signal, dafür leistet, dass Textverstehende dasjenige Wissen aktivieren können, das von ihnen

1 Zu nennen wären hier etwa die Begrenzung der Semantik auf wahrheitsfunktionale Aspekte, das Kompositionalitätsprinzip, wonach sich Satzbedeutungen aus Wortbedeutungen ohne weitere Hinzufügungen quasi „errechnen“ lassen, die Dominanz formallogischer über natürlich-sprachliche Aspekte und Annahmen (und die Hypothese, dass erstere letztere umfassend erklären könnten), aber auch das am Vorbild einer strikt differenziellen Phonologie orientierte Verständnis von Sprache als abstraktes System im Strukturalismus und die damit einhergehende Verkürzung von Bedeutungen auf Listen semantischer Merkmale, und vieles anderes mehr. 
aktivieren zu lassen die Textproduzenten beabsichtigt haben. Mit dieser Perspektive auf die Semantik und Textanalyse ist quasi automatisch verbunden, dass sie stets ein auch kulturwissenschaftlich operierendes Unterfangen ist und sein muss. Ich nenne dieses Unterfangen seit einigen Jahren „wissensanalytische“ oder eben „epistemologische“ Linguistik. ${ }^{2}$ Frame-Semantik könnte in meinen Augen eines der wichtigsten Modelle sein, um der Verwirklichung einer solchen epistemologischen Semantik oder Linguistik ein großes Stück näher zu kommen.

\section{Frame-Modelle}

Die Frame-Semantik, oder - allgemeiner betrachtet - die Frame-Theorie, ist kein einheitlicher Block, kein geschlossenes Modell, sondern zerfällt in unterschiedliche Konzeptionen mit Herkunft aus ganz verschiedenen Wissenschaften, die jeweils teilweise deutlich verschiedene Erkenntnisziele oder sogar Forschungsgegenstände haben. So hat etwa die Frame-Semantik von Charles Fillmore ${ }^{3}$ (und damit von FrameNet) - als einzige genuin linguistische Frame-Konzeption - ihre Wurzeln in ganz anders gearteten Überlegungen und Theoremen als die kognitionswissenschaftlichen Modelle etwa von Minsky (1974), Schank/Abelson (1977) und Barsalou (1992, 1993). Auch wenn Fillmore seine Konzeption seit Erscheinen von Minskys epochemachendem Aufsatz von 1974 mit dessen kognitionswissenschaftlicher Frame-Konzeption kurzgeschlossen hat, so ist doch nicht von der Hand zu weisen, dass sie ihre Wurzeln und theoretischen Bezugspunkte viel stärker in der Valenzgrammatik und der aus dieser in Fillmore (1968) abgeleiteten Theorie der „case-frames“ (Kasusrahmen) hatte und hat. Die Gemeinsamkeit dieser Konzeption mit dem von Minsky (1974) begründeten kognitionswissenschaftlichen Frame-Modell liegt vor allem in dem, was den Charme, die Besonderheit und den wesentlichen Kern der Frame-Theorien ausmacht: die Rede von Leerstellen und ihren Füllungen. Die Valenztheorie hatte diese Grundidee ihrerseits (zumindest implizit) metaphorisch aus der Chemie, genauer: aus der begrifflichen Unterscheidung zwischen der Bindungsfähigkeit von Atomen und den konkreten Bindungen in gegebenen Molekülstrukturen entlehnt. Auf dem Umweg über die ja zunächst auf Sätze und die Bindungsfähigkeit von zentralen Satz-Prädikaten in

2 Siehe dazu vertiefend und begründend Busse 2005 und 2008.

3 Siehe etwa Fillmore 1977a, 1982, 1985 und 2006. 
Form von Verben bezogene Grundidee der Valenzgrammatik und ihre semantische Erweiterung zur Kasus-Rahmen-Theorie wurde dieses Modell dann auf die inhaltlichen Strukturen von Begriffen übertragen - zumindest kann man das so sehen.

Dass die kognitionswissenschaftliche Frame-Theorie bei der Ausarbeitung ihrer Grundideen starke Anleihen bei Beobachtungen über Sprache gemacht hat, ist unübersehbar. In Bezug auf das Prinzip der Rekursivität wird dieses linguistische Erbe der kognitiven Frame-Theorie etwa von Barsalou auch ganz explizit erwähnt. ${ }^{4}$ Dass nicht nur bei Minsky und Schank/Abelson Beobachtungen über oder an Sprache einen wichtigen Kern ausmachen und zu den wichtigsten Belegen für die Wirksamkeit frame-spezifischer Mechanismen zählen, ist ebenso unabweisbar. Und schließlich darf nicht übersehen werden, dass selbst die Experimente, die den Gedächtnispsychologen Bartlett (1932) zu seiner von Minsky als Vorläufer der Frame-Theorie reklamierten Schema-Theorie inspiriert haben, weit überwiegend sprachgestützt bzw. sprachförmig waren.

All diese deutlichen Sprachbezüge und sprachtheoretischen Wurzeln auch der kognitionswissenschaftlichen Frame-Modelle dürfen und können aber nicht verdecken, dass genuin linguistische Frame-Konzeptionen, wie bei Fillmore, und die stärker oder rein kognitionswissenschaftlich motivierten Modelle, wie bei Minsky, Schank/Abelson oder Barsalou, doch erhebliche Unterschiede aufweisen. Insbesondere aus linguistischer Sichtweise treten diese Unterschiede deutlich zutage. Während die Bezugsgröße für die Frame-Idee bei Fillmore und FrameNet ganz eindeutig Sätze und um Verben oder Satz-Prädikate gruppierte Satz- und Wissensstrukturen sind, stellen etwa für das Frame-Modell von Barsalou eindeutig nominale Begriffe den bevorzugten wenn nicht einzigen Untersuchungsgegenstand und Modell-Bezugspunkt dar. Man könnte die Differenzen zwischen den Modellen vielleicht anhand der vor allem von John Searle (1969) stark gemachten grundlagentheoretischen Unterscheidung zwischen Referenz und Prädikation als den beiden kognitiven und sprachlichen Basisleistungen für Propositionen bzw. Aussagekerne oder das, was Logiker „Urteil“ oder Frege „Gedanken“ genannt haben, verdeutlichen. Fillmores und FrameNets Frame-Verständnis wäre

4 Dies, obwohl Barsalou sehr stark in der Tradition rein kognitionspsychologischer Begriffstheorien (prototype, exemplar and category theory) steht, die zumindest dem Anspruch nach zunächst unabhängig von Sprachverstehen sind und u. a. allgemeine Kategorisierungsexperimente betreffen. 
danach vor allem auf Prädikationen konzentriert, während Barsalou (und möglicherweise auch Minsky und andere Kognitionswissenschaftler) eher der Referenz dienende Nominale im Blick haben. ${ }^{5}$ Die inhaltlichen Unterschiede zwischen beiden Modellgruppen lassen sich, wie ich glaube, vor allem aus dieser Differenz im Untersuchungsgegenstand oder -interesse erklären.

Eine sprachtheoretisch reflektierte, linguistisch leistungsfähige und umfassende Frame-Theorie muss jedoch diese Differenzen überwinden, und - wenn dies möglich ist - zu einem übergreifenden theoretischen Standpunkt gelangen. Ziel muss es sein, ein integratives, linguistisch reflektiertes, die wichtigsten sprachtheoretischen Standards nicht unterschreitendes Frame-Modell zu konzipieren, das diejenigen sprachbezogenen Erkenntnisleistungen, die die bisherige frame-theoretisch fundierte Forschung bereits hervorgebracht hat, einzuschlieBen und zu erklären erlaubt. ${ }^{6}$

\section{Elemente, Ebenen und Aspekte von Frames}

Ich komme zu den für die Praxis einer frame-theoretisch reflektierten Semantik und Textanalyse wichtigsten Elementen, Ebenen oder Aspekten von Frames, die hier jedoch nur vergleichsweise knapp behandelt werden können. ${ }^{7}$

Der Begriff „Frame“ wird als epistemologischer bzw. kognitiver Grundbegriff verstanden. Das heißt für eine linguistische Anwendung der Frame-Theorie: Es ist davon auszugehen, dass Frame-Strukturen nicht nur bei Nomen oder Verben festzustellen sind, sondern bei allen Typen sprachlicher Zeichen (Lexemklassen), also auch bei Adjektiven, Adverbien, Präpositionen, Partikeln usw. Da Frames Grundstrukturen (-elemente) der Kognition bzw. des Wissens sind, und damit auf

5 Ich spreche hier von einer dominanten Sichtweise und dominanten, theorieprägenden Beispieltypen. Verbal beteuern indes beide Seiten, dass Ihr jeweiliges Modell auch zur Analyse des jeweils anderen Bedeutungstyps geeignet sei - ob diese Selbstbeschreibungen zutreffen, mag dahingestellt bleiben und müsste erst einmal überprüft werden. Große Zweifel daran sind angesichts der jeweils stark einseitig gefärbten Sichtweisen jedoch durchaus angebracht.

6 Die nachfolgenden Überlegungen basieren auf dem in Busse 2012 als Quintessenz aus einer Übersicht über die wichtigsten frame-theoretischen Ansätze und Aspekte formulierten und diese weiterentwickelnden „Arbeitsmodell“ der Frame-Semantik.

7 Da deren Zahl vergleichsweise hoch ist (in Busse 2012 waren es an die fünfzig, was die Länge des Kapitels zum dort vorgestellten frame-semantischen Arbeitsmodell wesentlich miterklärt). 
allen Ebenen von deren Beschreibung anzusetzen sind, ergibt sich zudem zwingend, dass verschiedene Ebenen und Typen von Frames (und Frame-Analyse) angesetzt werden müssen. Im Rahmen einer linguistischen Anwendung der Frametheorie sind es etwa folgende Dichotomien, die Ebenen-Differenzen kennzeichnen, die Frame-theoretisch und Frame-analytisch beachtet werden müssen: individuell(es Wissen) vs. sozial(es Wissen), Kurzzeitgedächtnis (bzw. Arbeitsgedächtnis) vs. Langzeitgedächtnis, token (Anwendungsfall, konkrete kognitive Aktivierung eines Frame-Elements oder einer Teil-Struktur) vs. type (Muster, allgemeine Regel), aktuell (bzw. „okkasionell“) vs. usuell (regelhaft), konkret vs. allgemein, Exemplar (das unter einen Frame fällt) vs. Kategorie.

Eine linguistische (semantische) Frame-Analyse erfasst mit der Annahme von „Frames“ Strukturen im Wissen, die hier vor allem als verstehensrelevantes bzw. verstehensermöglichendes Wissen ins Spiel kommen. Dabei kann nach übereinstimmender Auffassung fast aller Linguisten, die sich bisher Frame-analytisch betätigt haben, ${ }^{8}$ nicht strikt zwischen „sprachlichem Wissen“ und sogenanntem „Weltwissen“ (oder „enzyklopädischem Wissen“) unterschieden werden. Eine wichtige Interaktion (Wechselwirkung) zwischen „sprachlicher“ und allgemeinepistemischer Ebene liegt in der Tatsache, dass sprachliche Zeichen dazu dienen (dazu benutzt werden, die Funktion haben), Weltwissen in spezifischer Weise zu fokussieren. (Dies drückte Fillmore 1977a mit seinem Begriff der „Perspektive“ aus, die er z. B. bei den von ihm exemplarisch beschriebenen COMMERCIAL EVENTFrames durch Verben wie kaufen, verkaufen, bezahlen, kosten verwirklicht sieht.)

In der linguistischen (insbesondere der semantischen) Forschung können bisher zwei Spielarten von Frame-Begriffen und -Analyse unterschieden werden: (1) Frame-Analysen, die sich stärker auf nominale Lexeme konzentrieren (prototypisch: Barsalou 1992, aber auch Konerding 1993, Lönneker 2003), und (2) FrameAnalysen, die als Ausgangspunkt vorrangig verbale Lexeme im Auge haben (Fillmore und FrameNet). ${ }^{9}$ Beide Richtungen formulieren ihre Annahmen und Analyseschritte bislang stark aus der Perspektive nur der jeweils einen Wortart (bzw.

8 Fillmore 1985, Konerding 1993, Lönneker 2003, Ziem 2008, Busse 2012; siehe auch von kognitionspsychologischer Seite aus Barsalou 1993.

9 Blickt man nur auf die Zahlen der in FrameNet abgedeckten Daten könnte man diese These anzweifeln. Aktuell ist die Verteilung der dort beschriebenen Lexeme folgendermaßen: 5.278 Nomen, 5.068 Verben, 2.317 Adjektive, 429 andere Wortarten. Das ändert allerdings nichts daran, dass das dort benutzte Modell (wie auch alle „Paradebeispiele“ von Fillmore selbst zeigen) immer verbale 
Zeichenkategorie). Eine angemessene linguistische (semantische) Frame-Theorie und -Analyse kann aber nur eine solche sein, die sich von solchen einseitigen Blickwinkeln frei macht und ein Frame-Modell entwickelt, das für alle sprachlichen Zeichen gleichermaßen geeignet ist.

Frames sind geordnete Strukturen von Wissenselementen. Die innere Struktur von Frames ist in ihrer allgemeinsten Form als „slot-filler-Struktur“ („Leerstellen“ vs. „Füllungen“) beschrieben worden. Ich selbst würde eher die an Minsky angelehnten Bezeichnungen „terminals“ vs. „ascriptions“, bzw. „Anschlussstellen“ vs. „Zuschreibungen“ bevorzugen. Der Vorschlag Barsalous von „Attributen“ und „Werten“zu sprechen, stellt demgegenüber schon eine Konkretisierung und Eingrenzung dar, die stark am Modell nominaler Frames orientiert ist. So denkt man typischerweise eher an Gegenstände, wenn man Eigenschaften („Attribute“) zuschreibt, weniger an Handlungen, Geschehensabläufe, etc. Während Ausdrücke wie „slots/Leerstellen“ oder „fillers/Füllungen“ eher eine Anmutung des „Zwingenden“ vermitteln, drücken die von Minsky benutzten Ausdrücke wie „Anschlussstellen“ oder „Zuschreibungen“ besser die aktiven kognitiven (bzw. epistemischen, wissensaktivierenden) Leistungen (und letztlich auch Wahlmöglichkeiten) der Individuen aus, die diese kognitiven Aktivitäten vollziehen.

Entsprechend den verschiedenen Frame-Typen (in der Linguistik/Semantik orientiert etwa an unterschiedlichen Lexemklassen) ist es sinnvoll, auch bei den Slots bzw. Anschlussstellen verschiedene Typen zu unterscheiden. Für die häufigsten linguistisch-semantischen Anwendungen (Bezugsobjekte) der Frame-Theorie bzw. -forschung sind (mindestens) folgende zwei Typen von Slots anzusetzen: (1) „Attribute“ im Sinne von Barsalou, und (2) „Frame-Elemente“ im Sinne von Fillmore und FrameNet. (Ob noch weitere Typen von Slots unterschieden werden müssen, wäre zu diskutieren und wohl auch eine Sache der weiteren empirischen Forschung.) Dabei sind Fillmore-,Frame-Elemente“ - bezogen auf die Lexemklasse Verben - typischerweise Argumentstellen (Anschlussstellen für Komplemente bzw. „Ergänzungen“) im Sinne der Valenztheorie, aber auch Positionen für

Prädikate zum Ausgangspunkt hat, und Lexeme anderer Wortarten nur im Hinblick darauf betrachtet, was sie zum sog. „Verbalgeschehen“ beitragen. Das kann man nur eine „verbal zentrierte“ Frame-Theorie nennen. An der starken Dominanz von Nomen im Ansatz von Barsalou (1992) bestehen keinerlei Zweifel. 
den Anschluss von Informationen, wie sie typisch für Adjunkte („Angaben“, „adverbiale Bestimmungen“) sind (die „Mitspieler“ in der von einem Verb bezeichneten „Szene“, aber auch die „Begleitumstände“). Barsalou-„Attribute“ dagegen sind - bezogen auf die Lexemklasse Nomen - typischerweise Klassen von Eigenschaften, die an den Referenzobjekten einer Kategorie spezifiziert werden können (Größe, Farbe, Material usw.). ${ }^{10}$ Da jedoch auch das von Verben Bezeichnete (z. B. Handlungen) Eigenschaften haben kann (z. B. schlürfen die Eigenschaft mit Geräusch), ist der Frame-Elemente-Typ „Attribute“ keineswegs auf Nomen beschränkt, sondern es müssen bei Verben mindestens zwei der Frame-ElementeTypen angesetzt werden: Attribute und verbspezifische Frame-Elemente. - Umgekehrt können auch Nomen Argumente regieren, sodass auch bei ihnen mindestens zwei Slot-Typen realisiert sind (siehe etwa Vater, Kanzler, Architekt). ${ }^{11}$

Innerhalb der Gruppe der Eigenschafts-Frame-Elemente kann und sollte zwischen sogenannten strukturalen Frame-Elementen und funktionalen Frame-Elementen/Attributen differenziert werden. Strukturale Frame-Elemente beziehen sich typischerweise auf Attribute wie FARBE, FORM, GEWICHT bei physischen Entitäten (Dingen, Lebewesen, Personen); ORT, ZEIT, ZIEL usw. bei Handlungen, Ereignissen usw. Funktionale Frame-Elemente/Attribute werden in jüngster Zeit auch unter dem Begriff Affordanzen zusammengefasst. ${ }^{12}$ Affordanzen werden typischerweise bei Objekten, Dingen angenommen (meist bei Artefakten). Eine mögliche Arbeitsdefinition von Affordanzen wäre dann etwa: menschen-, benutzungs- und zweckbezogene funktionale Eigenschaften von Dingen (Bsp. Nagel,

${ }^{10}$ Der Ausdruck „typischerweise“ ist hier - zur Vermeidung von Missverständnissen - äußerst wichtig. Natürlich behaupten Fillmore wie Barsalou verbal, dass ihr jeweiliges Modell natürlich auch für die Analyse der jeweils nicht fokussierten Wortart geeignet sei.

${ }^{11}$ So ist ein Kanzler immer ein Kanzler von etwas (z. B. Kanzler der Bundesrepublik Deutschland, Kanzler der Universität Düsseldorf); ein Architekt ist immer ein Planer von etwas, wie als Rektionskomposita zu interpretierende Nominalkomposita wie Gartenarchitekt, Stadtarchitekt zeigen (letztere sind interessant, weil offenbar nur die Einsetzung eines Nicht-Standardwertes für das Objekt des Planens zur Bildung eines solchen Nominalkompositums führt, während zur Bezeichnung von Planern von Gebäuden, als Standardwert, ohne Zusatz nur als Architekt bezeichnet werden können).

${ }^{12}$ Der Begriff geht zurück auf den Kognitionspsychologen Gibson $(1977,1979)$. Eine vielzitierte Definition stammt von Norman $(1988,9)$ : „When used in this sense, the term affordance refers to the perceived and actual properties of the thing, primarily those fundamental properties that determine just how the thing could possibly be used. A chair affords (,is for ${ }^{6}$ ) support, and, therefore, affords sitting." 
Hammer, Schraubenzieher usw.). Eine differenziertere Unter-Typologie von Affordanzen muss vorerst jedoch noch Desiderat bleiben. Ich gehe davon aus, dass es neben den normalen Eigenschafts-Frame-Elementen noch einen eigenen Typ von Frame-Elementen gibt, die ich „Frame-Elemente mit Meta-Informationen“ oder kurz Meta-Frame-Elemente nenne, und die Informationen betreffen, die über anderen Informationen des Frames operieren und diese in bestimmten Hinsichten (wie etwa Sprechereinstellungen zum Inhalt, Bewertungen, Wissensmodi, Gewissheitsgrade, Fiktionalitätsannahme) zusätzlich charakterisieren oder modifizieren. ${ }^{13}$

Frames sind Strukturen aus Wissenselementen. Damit sind sie immer auch Strukturen aus Relationen zwischen Elementen. Den Relationen und ihren Typen kommt daher eine zentrale Funktion bei der Analyse von Frames zu. Dabei scheint es sinnvoll zu sein, verschiedene Typen von Relationen zu unterscheiden. Nach Busse (2012) könnte man u. a. folgende Typen von Relationen innerhalb von Frames unterscheiden:

(a) Attribut-Referenzpunkt-Relationen (gemeint ist die Relation zwischen einem Attribut und dem, wozu es Attribut ist; letzteres nennt Barsalou (1992) „Kategorie“, freilich ohne diesen Begriff jemals zu definieren oder zu erläutern);

(b) Wert-Attribut-Relationen (z. B. FARBE zu ROT, WEIß, BLOND, GOLDEN);

(c) Relationen zwischen Attributen gleichen Typs:

- Gruppen von Attributen, (z. B. verfügen Kategorien für Lebewesen über Attribute für GRÖßE, FARBE, GEWICHT);

- Strukturelle Invarianten (wenn bei einer bestimmten Kategorie bestimmte Attribute immer zusammen auftreten, z. B. bei Kategorien des Typs Vogel das Attribut HAUT immer nur zusammen mit dem Attribut FEDERN);

- Attribut-Constraints (ein Objekt, das über ein Attribut für OBERFLÄCHE verfügt, verfügt zwingend über ein Attribut FARBE); ${ }^{14}$

13 Als Beispiele können etwa Konnotationen (Schlampe z. B. transportiert immer eine Negativ-Bewertung der Sprecher) oder Modalpartikel (Peter kommt bestimmt heute ins Kino kommuniziert die Einschätzung eines Gewissheitsgrades von Sprecherseite) genannt werden.

14 Constraints sind nach Barsalou (1992) logisch oder sachlich zwingende Beziehungen zwischen einzelnen Frame-Elementen. Der Begriff Constraint ist in seiner Anwendung durch Barsalou jedoch 
(d) Relationen zwischen Attributen verschiedenen Typs: z. B.

- Relationen zwischen Meta-Attributen und deskriptiven Attributen (so gibt es z. B. - leider - für viele Menschen auf der Welt feste Relationen zwischen dem Attribut HAUTFARBE in Bezug auf andere Menschen und Meta-Frame-Elementen der Typen Bewertungen oder Emotionen);

- Relationen zwischen Aktanten-Attributen und Eigenschafts-Attributen;

(e) Relationen zwischen Werten (darunter v.a. Constraints im engeren Sinne). Frames können dann auch betrachtet werden als in sich in verschiedene Strukturebenen gegliedert. Neben der Ebene der Attribute und der Ebene der Werte ist mindestens die Ebene des Frame-Kerns oder Referenzpunkts zu nennen. Gemeint damit ist dasjenige, $z u$ dem ein Wissenselement Attribut (Eigenschaft) ist. Barsalou (1992) spricht hier immer von Kategorie, allerdings ohne diesen Terminus jemals zu definieren oder zu erläutern, behandelt ihn aber nicht als eigenes Element in der Wissens-Struktur eines Frames. Letzteres hat nach meiner Kenntnis bislang vor mir nur Birte Lönneker (2003) thematisiert. Da das „Objekt“ eines Frames ja epistemisch gesehen überhaupt erst durch die Zuschreibung von Eigenschaften (via Attributen und ihren Werten) als dieses Objekt konstituiert wird, wäre der von Barsalou (1992) hierfür verwendete Terminus Kategorie streng genommen verfehlt; präziser wäre es, abstrakt von einem (quasi „epistemisch leeren“) bloßen „Referenzpunkt“ zu sprechen, auf den sich die epistemischen Zuschreibungen von Attributen und Werten beziehen, die ihn überhaupt erst zu einem „Objekt“, zu einem „etwas“ in unserer (kognitiven/epistemischen) Welt machen. ${ }^{15}$

Neben dieser inneren strukturellen Gliederung von Frames in Attribute und Werte (und alle Arten von Relationen, die daraus abgeleitet werden können) ist ein zweiter, völlig anders gearteter Typus von innerer Struktur von Frames anzusetzen, der um das Begriffspaar type-token kreist. Eine sich darauf beziehende Unterscheidung könnte dabei die Unterscheidung von abstrakten Muster-Frames

alles andere als klar bestimmt. Auch seine Abgrenzung zu z. B. strukturellen Invarianten ist alles andere als eindeutig und einfach handhabbar.

${ }^{15}$ Hier schließen sich eine Menge komplexer Referenz-theoretischer Fragen an, für die Kognitivisten und Linguisten meistens nicht das nötige theoretische und begriffliche Rüstzeug haben; fündig würde man schon eher in philosophischen Arbeiten zur Referenz, etwa bei Kripke (1972), Strawson (1950) und (1952) u. a. 
und konkreten Exemplar-Frames sein. Streng genommen ist dies aber keine Unterscheidung innerhalb eines einzelnen Frames, sondern eine Unterscheidung, die sich auf verschiedene Typen oder Ebenen von Frames (als strikt zu unterscheiden von Ebenen innerhalb eines Frames) bezieht. Das Verhältnis beider Ebenen ist nicht nur eine Differenz zwischen einer Struktur aus leeren Slots (oder lediglich mit Standardwerten gefüllten Slots) und einer Struktur aus (mit konkreten Werten) gefüllten Slots. Vielmehr können Exemplar-Frames einem Muster-Frame zusätzliche Slots hinzufügen, wenn sie gehäuft (über eine größere Zahl von Exemplaren, oder in besonders salienten Exemplaren) auftreten.

Frames weisen weitere wichtige Merkmale auf, die nicht immer in der Literatur auch alle angemessen gewürdigt werden: Prototypikalität, Default-Werte, Konventionalität, Iterativität, Vernetzbarkeit. Frames sind prototypische Strukturen des Wissens (oder: Strukturen prototypischen Wissens). Diese Prototypikalität wurde bereits von Bartlett (1932) implizit herausgearbeitet, von Fillmore (1977a) und Minsky (1974) dann auch explizit erörtert. In der Prototypikalität der Frames schlägt sich die Sozialität bzw. Konventionalität des Wissens nieder. Prototypikalität kann sich in Frames so äußern, dass nicht jeder Slot bei jeder einzelnen kognitiven Aktualisierung des Frames relevant oder gegeben (aktiviert) sein muss. Der wichtigere (und wohl auch häufigere) Effekt der Prototypikalität der Frames ist, dass Slots prototypische Filler haben (können), die sog. Standard- oder Default-Werte. Es ist dabei das Wesen von Default-Werten, dass sie im Regelfall durch abweichende konkrete Werte ersetzt werden (können). Standard- oder Default-Werte entstehen durch hohe Rekurrenz (also Frequenz) oder durch große Nähe zu einem gesellschaftlichen, kulturell determinierten Ideal. Bei der Aktivierung von Defaults kann (möglicherweise auch im Wechsel) auf beide Faktoren rekurriert werden. Sowohl Rekurrenz als auch Idealität schaffen Präzedenzialität, die wiederum eine wichtige Voraussetzung der Standardisierung und Typisierung ist.

Ein wichtiges Merkmal von Frames (insbesondere von semantischen bzw. semantisch relevanten Frames) ist die Konventionalität dieser Strukturen des Wissens. Statt Konventionalität kann man auch sagen: gesellschaftliche Prägung (Sozialität). Auch wenn die kognitive Realisierung bzw. Aktualisierung von Frames immer individuell ist, so setzt ihre Kommunizierbarkeit immer auch ihre Konventionalität voraus. Ohne an dieser Stelle vertieft auf den komplexen Begriff (und 
die Problematik) der Konventionalität (sprachlicher Ausdrucksmittel) eingehen zu können, sei zumindest darauf hingewiesen, dass eine Konventionalität in wissensanalytischem Sinne auf die Erwartbarkeit des Vorhandenseins bestimmten Wissens bei den Kommunikations- bzw. Interaktionspartner hinausläuft). In diesem Sinne sind Wissensrahmen bzw. Frames Strukturen erwartbaren Wissens. Innerhalb der Erwartbarkeit können dann vermutlich Grade derselben unterschieden werden; eine dieser Graduierungen verläuft parallel mit der Unterscheidung von Wertebereichen (für Attribute/Leerstellen), Standardwerten (sog. Default-Werten) und konkreten, nicht-standardisierten und damit Einzelfallabhängigen Einzel-Werten. Wenn häufig vorkommende Werte zu Standardwerten werden, kann man von einer vollzogenen Konventionalisierung sprechen. Ebenso wenn Elemente aus der Werte-Ebene in die Ebene der fest mit einem Frame verbundenen Attribute aufrücken. Die Zusammenhänge zwischen solchen Ebenen der Konventionalisierung von bzw. innerhalb von Frames sind indes noch kaum erforscht (vgl. aber Ziem 2008, 198-229). ${ }^{16}$

Die Konventionalität ist mit einer anderen ihrer Kehrseiten, der Iteration bzw. Iterativität (Wiederholung und damit Bestätigung eines Musters in den einzelnen Akten der Anwendung dieses Musters) intern, d. h. begrifflich oder sachlogisch verbunden, indem Konventionalisierung als Prozess die Iteration der kognitiven Aktualisierung der theoretisch als Frames identifizierten Konstellationen von Wissenselementen notwendig voraussetzt. Iteration bewirkt die kognitive und epistemische Stabilisierung der Konstellationen von Relationen, als die die Frames zu gelten haben und trägt somit entscheidend zum „Fortleben“ der Frames bei.

Frames (auf der Eben allgemeiner gesellschaftlicher Wissensstrukturen, d. h. Muster oder Types) sind keine einfachen und geschlossenen Strukturen. Vielmehr muss mit erheblicher gesellschaftlicher Varianz im Grad der „Granulierung“ und Ausdifferenziertheit der Frames gerechnet werden. Aufgrund des allgemeinen Prinzips der Rekursivität sind Frames prinzipiell unendlich verfeinerbare Wissensstrukturen. Dies schlägt sich darin nieder, dass in gesellschaftlichen Domänen mit unterschiedlichem Wissensbedarf auch die Differenziertheit der Frames variiert (typischerweise bekannt als sog. Experten/Laien-Divergenz).

\footnotetext{
${ }^{16}$ Ein Teil dieser Prozesse wird in der Frame-Forschung unter dem Begriff des entrenchment (Verfestigung) diskutiert.
} 
Man kann davon ausgehen, dass Frames nicht nur intern strukturiert sind, sondern (in die andere Richtung) auch zu größeren Frame-Komplexen vernetzt sind. Unter dem Stichwort der Frame-Systeme oder Frame-Netze sind bislang vor allem Konzept-Taxonomien (sog. Ontologien) diskutiert worden. Über die Vernetzungen hinaus, die sich in solchen taxonomischen Wissens-Ordnungen ergeben, dürfen die assoziativen, häufig auf Analogiebildung, Wahrnehmung von Kontiguitäten, metaphorischen Übertragungen beruhenden Relationen zwischen Frames und Frame-Elementen in ihrer konstitutiven und strukturgebenden Wirkung für das gesellschaftliche und individuelle Wissen jedoch nicht unterschätzt werden. Vielleicht lassen sich folgende Typen von Frame-Systemen unterscheiden:

a. Frames als Frame-Systeme: Zunächst einmal ist jeder Frame (aufgrund des Prinzips der Rekursivität) immer zugleich auch ein Frame-System, da er aus Unter-Frames (z. B. im Falle eines instantiierten Frames aus Attribut-Frames und Werte-Frames) besteht. So enthält etwa ein schenken-Frame das Frame-Element AGENS, das nur von einem Unter-Frame MENSCH ausgefüllt werden kann, der selbst ja schon frame-strukturell gesehen hoch komplex ist.

b. Taxonomien: Taxonomien oder geordnete Begriffs-Systeme sind - frametheoretisch betrachtet - komplexe Makro-Frame-Systeme, die in zahlreiche Ebenen und Gruppen von Unter-Frame-Systemen zerfallen. (Ein typischer Fall dafür sind die bekannten Begriffs-Hierarchien.) Der für Taxonomien einschlägige Relationen-Typ ist die Relation der Hierarchie (Ober-Frame, Unter-Frame, siehe etwa die bekannten Relationen Oberbegriff/Unterbegriff). Ober-Frames in Taxonomien legen Frame-Elemente (Slots, Default-Werte) der Sub-Frames fest (entweder einzeln, als sog. „Vererbung“ von Frame-Elementen, typischerweise aber als Vererbung von ganzen Gruppen von Frame-Elementen). Beispiele dafür sind aus der Beschreibung von Begriffs- bzw. Wissensordnungen wie auch der sog. „semantischen Relationen“ bestens bekannt (z. B. Lebewesen, Tier, Säugetier, Kaniden, Hund, Dackel).

c. Kongruenz-Netzwerke: Im Unterschied zu den hierarchischen Relationen in Taxonomien beruhen Frame-Systeme im Sinne von Kongruenz-Netzwerken auf der Übereinstimmung einzelner Wissenselemente. Der für Kongruenz-Netzwerke einschlägige Relationen-Typ ist die Relation der Parallelität (oder genauer: Kon- 
gruenz bei Abwesenheit hierarchischer Relationen). ${ }^{17}$ Ein Spezialfall solcher Kongruenz-Netzwerke sind die bekannten „Wortfelder“ (etwa „stehendes Gewässer“ mit See, Teich, Tümpel, Lagune usw.).

d. Serialitäts-Netzwerke: Die einzelnen Frames in Serialitäts-Frame-Netzwerken können sich einzelne oder Gruppen von Frame-Elementen teilen (und tun dies in der Regel auch), sodass sie in dieser Hinsicht mit Kongruenz-Netzwerken übereinstimmen, doch ist das nicht zwingend. Der für Serialitäts-Netzwerke einschlägige Relationen-Typ ist die Relation der Folge-Beziehungen im weitesten Sinne. Serialitäts-Netzwerke treten in der Form von Ereignis-Frame-Systemen und Handlungs-Frame-Systemen (oder als Mischungen von beidem) auf. Der in der Literatur für einige Typen von Serialitäts-Netzwerken eingeführte Begriff ist der von Schank und Abelson (1977) ausführlich erläuterte und viel zitierte Skript-Begriff. Man kann dann evtl. noch verschiedene Aspekte oder Ebenen der Serialität unterschieden, wie etwa: Temporale Serialität, Kausal bedingte Serialität, ${ }^{18}$ Kulturell bedingte Serialität, Sprachliche Serialität. Am bekanntesten und häufigsten ist wohl der Typus der kausalen Serialität, den Schank und Abelson (1977) mit ihrem Skript-Modell am viel zitierten Beispiel Restaurant-Besuch beschrieben haben. Dieser lässt sich als kausal aufeinander aufbauende Aufeinanderfolge von Teilereignis-Frames beschreiben (Restaurant betreten, hinsetzen, Karte entgegennehmen, bestellen, speisen, zahlen, Restaurant verlassen). ${ }^{19}$ Ein Beispiel für eine nicht-kausale, rein temporale Serialität findet man etwa bei solchen Ereignis-Abfolgen wie Gottesdienst. ${ }^{20}$ Mit sprachlicher Serialität sind z. B. Textverläufe gemeint, wie sie für viele Textsorten typisch sind.

e. Assoziative Netzwerke: Der Typ von Frame-Systemen bzw. -Netzen mit der „schwächsten“ Form von Relationen sind die Assoziativen Netzwerke. Die für assoziative Netzwerke einschlägigen Relationen treten in unterschiedlicher Form auf. Als einschlägige Relationen-Typen können wohl mindestens die Relationen

\footnotetext{
${ }^{17}$ Sie entsprechen dem, was Minsky als „Ähnlichkeits-Netzwerke“ bezeichnet hat.

${ }^{18}$ Diese erste Unterscheidung ist insofern nicht ganz unproblematisch, als jede Kausale Serialität immer eine temporale Serialität umfasst bzw. voraussetzt. „Temporale Kausalität“ als eigener Serialitätstyp kann dann hier nur heißen: „temporale Abfolge ohne kausalen Zusammenhang“.

${ }^{19}$ Frame-theoretisch gesehen sind Skripts bestimmte spezielle Typen von Frames bzw. besser FrameFolgen. Dies haben sowohl Fillmore als auch Barsalou so gesehen.

${ }^{20}$ Rein temporale (d. h. nicht-kausale) Serialitäts-Netzwerke von Frames finden sich wohl besonders häufig bei kulturellen Ritualen; daher besteht hier häufig ein enger Zusammenhang mit dem Typus der „kulturellen Serialität“.
} 
der Kontiguität (Kopf - Lebewesen, Elektrogerät - Schaltknopf usw.), der Ähnlichkeit (berühmtes Auto von VW - Käfer), der partiellen Kongruenz (z. B. Isotopien) und der (prozeduralen) Korrelationen bzw. Ko-Okkurenzen (z. B. Schultafel - Kartenständer) genannt werden.

Insofern Frames auch als Strukturen der Erwartbarkeit aufgefasst werden können, sind die Elemente eines Frames graduell abstufbar nach dem Grad ihrer Erwartbarkeit. Hier ist es aber (aus Gründen, die etwas mit den Strategien der Informationsvermittlung zu tun haben, insbesondere solchen Strategien, die in sprachlichen Regeln verfestigt sind) notwendig, zwischen drei Formen (Typen, Ebenen) der Erwartbarkeit zu unterscheiden, die ich in einem heuristischen Vorgriff auf präzisere Analysen vorerst präsuppositive, parasuppositive und konstitutive Erwartbarkeit nennen möchte. Die präsuppositive Erwartbarkeit bezieht sich auf all solche Wissenselemente, die dem in einem Frame organisierten Wissen (oder einzelnen seiner Elemente) logisch oder epistemisch vorgängig sind, sei es, dass sie aufgrund taxonomischer Relationen in den Wissenselementen des Frames epistemisch oder logisch „enthalten“ sind, sei es, dass sie in Kausal-Relationen (oder ihrer Umkehrung als Konditional-Relationen) notwendig als gegeben vorauszusetzen sind. (Z. B. setzt Die Tür schlug $z u$ voraus, dass vorher Die Tür stand auf als gegeben galt.)

Zur Gruppe (Ebene) der parasuppositiven Erwartbarkeit zählen all solche Wissenselemente, die dem in einem Frame organisierten Wissen (oder einzelnen seiner Elemente) solche Aspekte hinzufügen, die in kommunikativen Akten der Informationsvermittlung thematisch werden können, aber nicht müssen. (Dass Gegenstände, Personen, Ereignisse, Handlungen ORTE haben und in Bezug auf ZEITPUNKTE oder ZEITRÄUME spezifiziert sind, kann thematisch werden; aber in vielen - vielleicht den meisten - Fällen ihrer aktiven kognitiven Prozessierung ist dies nicht notwendig.) Von beiden Gruppen (Ebenen) ist die Gruppe (Ebene) derjenigen Wissenselemente abzugrenzen, die zur Kategorie der konstitutiven Erwartbarkeit zu rechnen sind. Konstitutiv erwartbare Frame-Elemente sind per se immer thematisch, auch wenn sie nicht immer explizit verbalisiert werden müssen (sie haben so eine Art „inhärente oder eingebaute Thematizität“). Nur in Bezug auf diese Gruppe (oder Ebene) macht Fillmores Begriff der „Null-Instantiierung“ von Frame-Elementen überhaupt einen Sinn. So ist in Peter fuhr in die Stadt der Aktanten-Slot für FAHRZEUG im Sinne des Ansatzes von Fillmore und FrameNet „null- 
instantiiert“, aber natürlich implizit „mitgedacht" und damit im Sinne des von mir vorgeschlagenen Begriffs „konstitutiv erwartbar“.

Soweit die wichtigsten Merkmale und Aspekte von Frames und Frame-Strukturen.

\section{Zur Praxis einer linguistischen Frame-Analyse}

Abschließend noch ein paar Bemerkungen zu einigen praktischen Aspekten einer Frame-Analyse in der Linguistik. Jeder praktischen Frame-Analyse oder jedem Versuch einer linguistisch motivierten Frame-Beschreibung stellt sich die Frage nach den Informationen, die in einer Frame-Beschreibung darzustellen sind. Vor jeder Analyse ist daher festzustellen, welche Aspekte und Elemente von Frames in einer schematischen Gesamtdarstellung eines Frames überhaupt erfasst werden müssen oder sollten. Der Hinweis auf den schematischen Charakter einer Frame-Darstellung soll verdeutlichen, dass grundsätzlich auch ganz andere Arten von Frame-Darstellungen denkbar und möglicherweise sinnvoll sind. So ist nicht ausgeschlossen, dass gerade bei der Darstellung sehr komplexer Frames oder Frame-Strukturen in tiefensemantischen (z. B. kulturwissenschaftlich-epistemologisch motivierten) Analysen nur die Form einer Monographie (oder einer umfassenderen Darstellung mit Kapitelumfang) ausreichend ist, um alle wichtigen Aspekte eines Frames in ihrer Gesamtheit zu erfassen. Ich gehe davon aus, dass eine umfassende Frame-Darstellung auf folgende Aspekte achten sollte; dabei handelt es sich bei der (nicht zwingend als abgeschlossen zu betrachtenden) Liste um eine heuristische Sammlung von Aspekten, die sich aus einer Gesamtschau der einschlägigen, für linguistische Zwecke interessanten Literatur zur FrameAnalyse ergibt:

In einer Frame-Darstellung zu erfassende Typen von Informationen:

Frame-Name (Frame-Kern, „Kategorie“) [FN]

Frame-Elemente (Attribute, Slots) FE-Name

FE-Typ

Relationen-Typ FE zu FN

Wertebereich

Standardwerte

Zentralität, Salienz der FE 
Überlegungen zu einem integrativen Frame-Modell: Elemente, Ebenen, Aspekte

$\begin{array}{ll} & \text { Epistemischer Status } \\ & \text { Zugehörigkeit zu einer FE-Gruppe } \\ \text { Frame-Elemente-Gruppen [FEG] } & \text { FEG-Typ } \\ & \text { Relationen-Typ in der FEG } \\ \text { Constraints } & \text { Constraints zwischen Frame-Elementen } \\ & \text { Constraints zwischen Werten/Fillern } \\ & \text { Constraints zwischen FE und Werten } \\ \text { Frame-zu-Frame-Beziehungen } & \text { Vererbung, Ober-Frame(s) } \\ & \text { Sub-Frames } \\ & \text { Relationen-Typ(en) der F-zu-F-Bez. }\end{array}$

Hierzu ein paar Erläuterungen:

Der Frame-Name (auch Frame-Kern oder Kategorie genannt) stellt als eine Art Archi-Lexem den Titel des Frames dar und markiert, dass es hier ein (epistemisches) „Etwas“ gibt, auf das sich die nachfolgend spezifizierten Frame-Elemente, Relationen und Aspekte beziehen.

Frame-Elemente werden hier in einem etwas enger gefassten Gebrauch dieses Terminus die Anschlussstellen/Slots/Attribute eines Frames genannt, also diejenigen Elemente, die einen Frame als diesen Frame definieren bzw. ausmachen oder konstituieren. Zwar gehören auch die Werte bzw. Filler zu den Frame-Elementen in einem weiter gefassten Sinn, doch werden sie nur vermittels der Slots/ Attribute an einen Frame angeschlossen und spielen etwa in einer type-bezogenen Frame-Darstellung nur eine untergeordnete Rolle. Für eine solche Darstellung reicht es, den Wertebereich anzugeben. Nur in token-Frame-Darstellungen bekommen die Werte zwingend einen eigenen Platz. Frame-Elemente werden durch einen Frame-Elemente-Namen gekennzeichnet. (Die Wahl eines solchen Namens ist aller Erfahrung nach nicht immer ganz einfach, da es sich hier um abstrakte Kategorien handeln kann, für die die jeweilige Beschreibungssprache kein Lexem vorhält. Es kann daher sein, dass man zu einer sprachlich komplexeren Umschreibung greifen muss.)

$\mathrm{Zu}$ jedem festgestellten Frame-Element muss/sollte immer der Frame-Elemente-Typ (Aktanten-FE, Eigenschafts-FE, funktionale FE bzw. Affordanzen, Meta-FE) angegeben werden. 
Außerdem sollte der Relationen-Typ für die Relation des Frame-Elements zum Frame-Kern angegeben werden. (Hierzu gibt es in der bisherigen Frame-Literatur nur wenige Hinweise und kaum Vorbilder.)

Eine der wichtigsten Informationen zu einem Frame ist die Angabe des jeweiligen Wertebereichs und der Standard-Werte für jedes Frame-Element. Die Darstellung von Wertebereichen ist vor allem für type-Frame-Darstellungen relevant und kann bei token-Frame-Darstellungen auch (je nach Darstellungsinteresse) unterbleiben. Bisher werden Wertebereiche in kaum einer Frame-Darstellung als solche explizit charakterisiert. Meist findet man stattdessen exemplarische Auflistungen typischer Füllwerte. Zur Darstellung des Wertebereichs gehört auch die Angabe des Typs des Wertebereichs. Für die meisten Frame-Elemente stellt die Angabe von prototypischen Standard-Werten eine zentrale Information dar. Zur Charakterisierung des Wertebereichs eines FE kann auch die Angabe gehören, ob das FE überhaupt mit einem konkreten Wert belegt werden muss, oder ob im Regelfall der Standardwert eingesetzt wird.

Der zuletzt genannte Aspekt berührt sich mit der Frage der Salienz oder Zentralität des jeweiligen Frame-Elements für den Frame. Nicht alle Frame-Elemente in einem Frame sind gleichrangig oder gleichermaßen kognitiv bzw. epistemisch zentral; solche Unterschiede müssen aber in einer Frame-Darstellung markiert werden, wenn diese das Frame-relevante Wissen adäquat wiedergeben soll. Vermutlich werden in vielen Fällen der kognitiven Aktualisierung von Frames (den Frame-Instantiierungen oder token-Frames) bestimmte Frame-Elemente des typeFrames gar nicht aktiv prozessiert. Soweit möglich sollten solche Informationen in der Frame-Darstellung erfasst werden.

Ob der oben genannte epistemische Status der Frame-Elemente einen eigenen Typ von Informationen darstellt, der in einer Frame-Beschreibung erfasst werden sollte, ist noch nicht ausgemacht, da es dazu bislang kaum Forschungen oder auch nur Überlegungen gibt. Möglicherweise lassen sich Informationen zum epistemischen Status von Frame-Elementen aufteilen auf die Beschreibungskategorien (bzw. -Schritte) Salienz (etwa was den Aspekt der epistemischen Zwingendheit eines Frame-Elements angeht) und Frame-Elemente-Typ (etwa was den Aspekt der Wahrscheinlichkeit angeht). 
Ein letzter wichtiger Typ von Informationen zu den einzelnen Frame-Elementen ist die Angabe der Zugehörigkeit zu einer Frame-Elemente-Gruppe (den strukturellen Invarianten in der Terminologie Barsalous), soweit eine solche im gegebenen Frame vorliegt.

Die Markierung von Frame-Elemente-Gruppen (strukturellen Invarianten) in Frames ist eine weitere wichtige Art von Informationen, die neben der Charakterisierung der Frame-Elemente selbst unbedingt zu einer adäquaten Frame-Beschreibung dazugehört. Zu der Darstellung der Frame-Elemente-Gruppen (FEG) gehört die Darstellung des FEG-Typs. (Eine Typologie dafür muss freilich noch ausgearbeitet werden.) Ebenfalls gehört dazu die Charakterisierung des Typs der Relationen innerhalb der jeweiligen FEG.

Ein weiterer sehr wichtiger Typ von Angaben in einer Frame-Darstellung auf oberster Ebene ist die Darstellung der in einem Frame wirksamen Constraints (im Sinne Barsalous). Zu unterscheiden sind dabei Constraints zwischen Frame-Elementen, Constraints zwischen Werten/Fillern (verschiedener FE) und Constraints zwischen FE und Werten (anderer FE). Möglicherweise muss noch eine Angabe zum Typ des Constraints hinzukommen (falls eine solche Typologie in sinnvoller Weise entwickelt werden kann).

Eine letzte Gruppe von sehr zentralen Elementen in jeder Frame-Beschreibung sind die Frame-zu-Frame-Relationen. Hier sind insbesondere die Vererbungs-Relationen und die Sub-Frame-Relationen zu nennen. Auf jeden Fall muss jede Framezu-Frame-Relation hinsichtlich ihres Relationen-Typs spezifiziert werden.

Anwendungsmöglichkeiten der Frame-Analyse:

Schließlich stellt sich die Frage nach den Anwendungsmöglichkeiten der FrameAnalyse in der Linguistik. Fillmore hat in seinen Schriften mehrfach an verschiedenen Stellen beeindruckende Listen der Leistungsfähigkeit eines linguistischen Frame-Modells publiziert. ${ }^{21}$ Als die wichtigsten Anwendungsmöglichkeiten seien hier (im Anschluss an Busse 2012, 742ff.) folgende genannt:

- Frame-Analyse von Lexemen und Wortbedeutungen

- Frame-Analyse von Morphemkombinationen und Wortbildungen

- Frames für Begriffstypen und Wortarten

- Frame-Analyse von Textwörtern im Kontext

${ }^{21}$ So in Fillmore (1977a, 129ff.), Fillmore (1982, 124ff.); vgl. dazu Busse (2012, 132ff.). 
- Frame-Analyse von Satzbedeutungen

- Frames in der Textanalyse und Verstehenstheorie

- Frames in der Analyse von Metaphern

- Frames in der Analyse von Präsuppositionen und Implikaturen

- Frame-Analyse von Bedeutungs- und Sprachwandel

Bevorzugtes Objekt von linguistischen Frame-Analysen waren bisher freilich vor allem Wörter. Nur gelegentlich wurden auch Sätze analysiert. Die Methoden einer satzsemantischen und textanalytischen linguistischen Frame-Analyse sind daher bislang noch vergleichsweise wenig ausgearbeitet. Dies erstaunt, wenn man berücksichtigt, dass insbesondere die Frame-Idee von Fillmore stark mit seinem Ziel einer eindeutig textsemantisch ausgerichteten understanding-semantics verknüpft war und auch Bartlett und Minsky bereits auf die Funktion von Frames zum Verstehen von Geschichten verwiesen haben. Auch ich kann für eine tiefensemantisch ausgelegte linguistisch motivierte Frame-Analyse nicht die umfassende und fertige Methodik liefern, sondern nur betonen, dass eine semantisch orientierte Textanalyse durch eine Berücksichtigung der hier vorgestellten frame-analytischen Gesichtspunkte und Untersuchungsziele m. E. eindeutig gewinnt.

\section{Leistungen und Grenzen einer Frame-Semantik}

Die empirische (linguistische) Forschung zur Frame-Semantik ist noch zu jung und zu selten umgesetzt worden - im Sinne einer systematischen, das gesamte Spektrum der theoretischen Modelle und Möglichkeiten umsetzenden umfassenden Forschung -, als dass eine abschließende Einschätzung ihrer Leistungspotenziale und ihrer Grenzen bereits möglich wäre. Von Linguisten wie Fillmore (und Autoren des FrameNet-Verbunds) sind ebenso wie von Kognitionswissenschaftlern wie Minsky und Barsalou beeindruckende Listen erstellt worden darüber, was alles mit Hilfe eines (jeweils unterschiedlich ausfallenden) Frame-Modells im Bereich der Gegenstände der Sprachforschung im weitesten Sinne erforscht werden könne. Von Verben, Nomen und Sätzen angefangen, über kognitive Konzepte, Texte, Morpheme, Metaphern, Anaphern, Präsuppositionen bis hin zu Prä- 
positionen und Konjunktionen sind fast alle Gegenstände im Umfeld der Linguistik schon einmal als mögliches Anwendungsobjekt einer Frame-Forschung genannt worden. So weit die ambitionierte Programmatik.

Es steht aber zu vermuten, dass es nicht so sein wird, dass alle genannten Phänomene gleichermaßen gut (oder überhaupt) mit demselben Frame-Modell analysiert werden können. So fragt sich z. B., ob die häufig von Fillmore angesprochenen „Hintergrund-Frames“ (oder „Szenen“) mit einem Barsalou-Modell der Frames überhaupt angemessen oder vollständig erfasst werden können. Für zahlreiche von Fillmores Parade-Beispielen (wie Waise, Witwe, Junggeselle, Vegetarier, an Land, auf dem Boden, vgl. Fillmore 1977a, 72; Fillmore 1978, 165; Fillmore 1976b, 26; Fillmore 1975c, 139) gilt, dass dasjenige Wissen, auf dessen Rolle für ein adäquates Verstehen er mit diesen Beispielen anspielen will, teilweise so komplex und voraussetzungsvoll ist, dass eine angemessene Paraphrase möglicherweise jeweils zusätzlich eine größere Zahl von Prädikationen einführen muss, die jeweils für sich in allen ihren Elementen Frame-semantisch analysiert und bestimmt werden müssten. Dadurch käme man schnell zu einer ziemlich komplexen Beschreibung, wenn man z. B. ein Modell des Barsalou-Typs systematisch darauf anwenden wollte. Eine detaillierte Frame-Analyse größerer sprachlicher Komplexe (etwa ganzer Texte oder längerer Textabschnitte) scheint ${ }^{22}$ erst recht außerhalb des Machbaren und Sinnvollen zu liegen (weil die Ergebnisse so komplex würden, dass sie vollends unübersichtlich und kaum noch lesbar und benutzbar wären).

Es wäre also falsch, in der Frame-Theorie ein Allheilmittel für alle linguistischen (oder semantischen) Fragestellungen und Untersuchungsziele zu sehen. Die Frame-Theorie ist dort stark, wo sie in die erkennbaren Lücken älterer linguistischer Programme (wie der Merkmalsemantik, der Logischen Semantik, der wort-isolierenden lexikalischen Semantik, der logik-fundierten kompositionalistischen Satzsemantik, der wort- und begriffs-isolierenden historischen Semantik) stößt. Genauer gesagt: Überall dort, wo der Umfang, die Komplexität, die Subtilität und die Ausdifferenziertheit des verstehensrelevanten bzw. sprachrelevanten

22 Zumindest wenn man nicht bereit ist, so reduktionistisch vorzugehen, wie dies in einigen Beispielen im Rahmen und nach dem Vorbild des FrameNet-Projektverbunds der Fall ist, sondern eine wissensanalytisch umfassende und detaillierte Analyse anstrebt, wie sie in diesem Papier und in Busse (2012) angedacht ist. 
Wissens in den älteren Modellen sträflich unterschätzt wurde. Vor allem hier kann sie ihre besondere Leistungsfähigkeit entfalten und ist anderen Ansätzen überlegen. Die Grenzen ihrer Möglichkeiten werden (und können) aber letztlich erst dann sichtbar werden, wenn diese Möglichkeiten in empirischen Analysen unterschiedlichster Form auf breitem Felde umgesetzt und praktisch erprobt wurden. Von diesem Punkt sind wir momentan jedoch noch sehr weit entfernt.

\section{Literatur}

Barsalou, Lawrence W. (1992): Frames, concepts, and conceptual fields. In: Adrienne Lehrer / Eva. F. Kittay (eds.): Frames, Fields, and Contrasts. Hillsdale, NJ: Lawrence Erlbaum Associates, 21-71.

Barsalou, Lawrence W. (1993): Flexibility, Structure, and Linguistic Vagary in Concepts: Manifestations of a Compositional System of Perceptual Symbols. In: Collins, A. C. / Gathercole, S. E. / Conway, M. A. (eds.): Theories of Memory. Hillsdale, NJ: Lawrence Erlbaum Associates, 29-101.

Bartlett, Frederick C. (1932): Remembering: A Study in Experimental and Social Psychology. Cambridge: Cambridge University Press.

Busse, Dietrich (2005): Architekturen des Wissens. Zum Zusammenhang von Semantik und Epistemologie. In: Ernst Müller (Hrsg.): Begriffsgeschichte im Umbruch. (Archiv für Begriffsgeschichte, Sonderheft 2004) Hamburg: Felix Meiner, 85-99.

Busse, Dietrich (2008): Linguistische Epistemologie. Zur Konvergenz von kognitiver und kulturwissenschaftlicher Semantik am Beispiel von Begriffsgeschichte, Diskursanalyse und Frame-Semantik. In: Heidrun Kämper (Hrsg.): Sprache - Kognition - Kultur. Sprache zwischen mentaler Struktur und kultureller Prägung. (= Jahrbuch 2007 des Instituts für deutsche Sprache) Berlin / New York: de Gruyter, 73-114.

Busse, Dietrich (2012): Frame-Semantik - Ein Kompendium. Berlin / Boston: de Gruyter.

Fillmore, Charles J. (1968): The Case for Case. In: Emmon Bach / Robert T. Harms (eds.): Universals in Linguistic Theory. New York: Holt, Rinehart \& Winston 1968, 1-88. [Teilabdruck in: René Dirven / Günter A. Radden (eds.): Fillmore’s Case Grammar. A Reader. Heidelberg: Groos 1987, 21-34. - Dt. Übers. in: 
Werner Abraham (Hrsg.): Kasustheorie. Frankfurt a. M.: Athenäum 1971, 1-118.]

Fillmore, Charles J. (1975): The Future of Semantics. In: Robert Austerlitz (ed.): The Scope of American Linguistics. Lisse: Peter de Ridder Press, 135-157.

Fillmore, Charles J. (1976): The need for frame semantics in linguistics. In: Hans Karlgren (ed.): Statistical Methods in Linguistics 12, 5-29.

Fillmore, Charles J. (1977a): Scenes-and-Frames Semantics. In: Antonio Zampolli (ed.): Linguistic Structures Processing. Vol. 5. Amsterdam / New York / Oxford: North Holland, 55-81. [Teilabdruck in: René Dirven / Günter A. Radden (eds.): Fillmore's Case Grammar. A Reader. Heidelberg: Groos 1987, 79-88.]

Fillmore, Charles J. (1977b): Topics in Lexical Semantics. In: Roger W. Cole (ed.): Current Issues in Linguistic Theory. Bloomington / London: Indiana University Press, 76-138. [Teilabdruck in: René Dirven / Günter A. Radden (eds.): Fillmore's Case Grammar. A Reader. Heidelberg: Groos 1987, 89-98.]

Fillmore, Charles J. (1978): On the organization of semantic information in the lexicon. In: Donka Farkas / Wesley M. Jacobsen / Karol W. Todrys (eds.): Papers from the Parasession on the Lexicon. Chicago: The Chicago Linguistic Society, 148-173.

Fillmore, Charles J. (1982): Frame Semantics. In: The Linguistic Society of Korea (ed.): Linguistics in The Morning Calm. Seoul: Hanshin Publishing Corp., 111-137.

Fillmore, Charles J. (1985): Frames and the Semantics of Understanding. In: Quaderni di Semantica 6, 222-254.

Fillmore, Charles (2006): Frame Semantics. In: Keith Brown (ed.): Encyclopedia of Language and Linguistics. $2^{\text {nd }}$ Edition. Amsterdam: Elsevier, 613-620.

Gibson, James J. (1977): The theory of affordances. In: Robert E. Shaw / John Bransford (eds.): Perceiving, acting, and knowing. Hillsdale, NJ: Lawrence Erlbaum Associates, 67-82.

Gibson, James J. (1979): The ecological approach to visual perception. Boston: Houghton Mifflin.

Konerding, Klaus-Peter (1993a): Frames und lexikalisches Bedeutungswissen. Untersuchungen zur linguistischen Grundlegung einer Frametheorie und zu ihrer Anwendung in der Lexikographie. Tübingen: Niemeyer. 
Kripke, Saul A. (1972): Naming and Necessity. In: Donald Davidson / Gilbert Harman (eds.): Semantics of Natural Language. Dordrecht-Holland, 253-355 und 763-769. [dt.: Name und Notwendigkeit. Frankfurt a. M.: Suhrkamp.]

Lönneker, Birte (2003): Konzeptframes und Relationen. Extraktion, Annotation und Analyse französischer Corpora aus dem World Wide Web. Berlin: Akademische Verlagsgesellschaft AKA.

Minsky, Marvin (1974): A Framework for Representing Knowledge. In: Artificial Intelligence Memo No. 306, MIT Artificial Intelligence Laboratory. [Abgedruckt in: Patrick H. Winston (ed.): The Psychology of Computer Vision. New York: McGraw-Hill, 1975, 211-277. - Auszug abgedruckt in: Dieter Metzing (ed.): Frame Conceptions and Text Understanding. Berlin / New York: de Gruyter, 1980, 1-25. - Dt. in: Dieter Münch (Hrsg.): Kognitionswissenschaft. Grundlagen, Probleme, Perspektiven. Frankfurt a. M.: Suhrkamp, 1992, 92-133].

Norman, Donald A. (1988): The Psychology of Everyday Things. New York: Basic Books.

Schank, Roger C. / Abelson, Robert P. (1977): Scripts, Plans, Goals and Understanding: An Inquiry into Human Knowledge Structures. Hillsdale: Lawrence Erlbaum.

Searle, John R. (1992): Sprechakte. Ein sprachphilosophischer Essay. Frankfurt am Main: Suhrkamp (zuerst 1969).

Strawson, Peter F. (1950): On referring. In: Mind, New Series, Vol. 59, No. 235. (Jul., 1950), 320-344. [Wiederabdruck in: Aloysius P. Martinich (ed.): The Philosophy of Language, $4^{\text {th }}$ edition. Oxford: Oxford University Press 2001, 20ff.] Strawson, Peter F. (1952): Introduction to Logical Theory. London: Methuen.

Ziem, Alexander (2008): Frames und sprachliches Wissen. Kognitive Aspekte der semantischen Kompetenz. Berlin / New York: de Gruyter. 